\title{
INVESTIGACIÓN PARA LA PAZ: PERSPECTIVA DE PAZ DESDE LA PRIMERA INFANCIA Y SUS AGENTES RELACIONALES*
}

\author{
RESEARCH FOR PEACE: PERSPECTIVE OF PEACE FROM EARLY \\ CHILDHOOD AND ITS RELATIONAL AGENTS
}

Estefanía Aristizábal-Ramírez**

\section{Resumen}

Objetivo. Ampliar los horizontes reflexivos en torno a una crianza para la paz, que configura escenarios en tiempos de posacuerdo/posconflicto para los niños y las niñas de la primera infancia, a partir del agenciamiento social de las docentes, padres/madres o cuidadores en el municipio de Marmato, Caldas. Metodología. El enfoque cualitativo que se utilizó es el crítico social. Resultado. Emergen cuatro potenciales del desarrollo humano en la primera infancia, el potencial del juego, el potencial de las voces de los niños y las niñas, el potencial del cuerpo y el potencial reflexivo como un aporte metodológico a la investigación para la paz. Conclusión. Para configurar escenarios y espacios del buen trato a partir de la cultura de paz en la primera infancia, es necesario la corresponsabilidad entre las familias y agentes educativos y un diálogo permanente con la política pública de la primera infancia.

Palabras clave: primera infancia, educación de la primera infancia, familia, paz.

\begin{abstract}
Objective. To expand the reflective horizons around a parenting for peace which configures scenarios in post-agreement/post-conflict times for early childhood children, starting with the social agency of teachers, parents/care attendants in the municipality of Marmato, Caldas. Methodology. The qualitative approach used was the social critic approach. Results. Four potentials of human development emerge in early childhood: the potential of the game, the potential of children's voices, the potential of the body and the reflective potential as a methodological contribution to research for peace. Conclusion. To configure scenarios and spaces of good treatment based on the culture of peace in early childhood, it is necessary to share responsibility between families and educational agents and a permanent dialogue with the early childhood public policy.
\end{abstract}

Key words: Early childhood, early childhood education, family, peace.

\footnotetext{
*Artículo de resultado de investigación, elaborado de la tesis de maestría "Investigación social: fortalecimiento en capacidades de las agentes educativas y familias en el eje cafetero"

** Centro de Estudios Avanzados en Niñez y Juventud-CINDE. Manizales, Colombia. E-mail: earistizabal@cinde.org.co

(D) orcid.org/0000-0002-0830-1444 Google Scholar
} 
Investigación para la paz: perspectiva de paz desde la primera infancia y sus agentes relacionales

\section{Introducción}

El interés general de esta investigación realizada en el marco del programa de Colciencias “Sentidos y Prácticas Políticas de Niños, Niñas y Jóvenes en Contextos de Vulnerabilidad en el Eje Cafetero, Antioquia y Bogotá: Un Camino Posible de Consolidación de la Democracia, la Paz y la Reconciliación mediante Procesos de Formación Ciudadana”, está enfocada a fortalecer las competencias parentales para la crianza orientadas hacia la promoción de conductas prosociales en niños y niñas de 3 a 5 años del eje cafetero ${ }^{1}$ en condiciones de riesgo y vulnerabilidad social, mediante el diseño e implementación de una intervención formativa diferenciada según el lugar, situación, etnia y género a partir del establecimiento de una línea de base de las competencias parentales, la prosocialidad infantil y el conocimiento ecoetnoantropológico de la familia y su contexto.

En respuesta a lo anterior, el eje problemático de la investigación es: ¿De qué manera el desarrollo de las competencias parentales en la familia potencian las habilidades prosociales en niños y niñas en edades de 4 a 7 años del municipio de Marmato, Caldas, que puedan aportar a la construcción de una cultura de paz?

El estudio se despliega con una apuesta cualitativa de intervención y de formación a partir del diplomado "Crianza para la Paz", cuyo objetivo principal es fortalecer en las agentes educativas, padres/madres o cuidadores la capacidad de agenciamiento de los potenciales del desarrollo humano en los niños y las niñas de primera infancia.

A través de estrategias lúdico-creativas se resignificaron sentidos y prácticas en torno al potencial del juego como estimulador permanente y potenciador de las habilidades prosociales como la cooperación, el altruismo, la empatía, los procesos asociativos entre experiencias ajenas y las propias de emoción similar.

El potencial del cuerpo es uno de los principales aspectos al considerar la relación que el niño y la niña establece consigo mismo, en interacción con los otros y con el entorno que lo rodea ya que, en esta etapa de desarrollo, emergen una cantidad de sensaciones y emociones que son experimentadas a través del cuerpo, pues se capta todo lo que existe a su alrededor; por lo tanto, es necesario reconocer a los niños y las niñas como sujetos de deseos, con capacidad de dar y recibir afecto, seguros de sí mismos, seres que se autoafirman en la relación con los otros pares (aspectos que permiten el desarrollo de la autoestima y la autonomía) de tal modo que,

\footnotetext{
${ }^{1}$ El Eje Cafetero es una región geográfica, cultural, económica y ecológica de Colombia ubicada en Caldas, Risaralda, Quindío, la región norte del Valle del Cauca, y el noroccidente del Tolima y las ciudades capitales de los tres primeros departamentos mencionados (Manizales, Pereira y Armenia, respectivamente), siendo Pereira la ciudad más poblada de la región, con su área metropolitana de Centro Occidente, con más de 700.000 habitantes. Esta región fue una notable productora de caucho a comienzos del siglo XX, pero luego se dedicó al café.
} 
a partir de esos nuevos lugares de las emociones, es primordial concebirlos desde la dimensión afectiva y ética por el cuidado de sí y del otro.

El potencial de la voz resulta fundamental para fortalecer procesos de diálogo entre el niño, la niña y sus agentes relacionales (adultos significativos), basándose en la intención de una comunicación asertiva y afectiva libre de dominio y autoridad por parte del adulto, y se instaura el lenguaje como acción hacia la comunicación orientada a la construcción de acuerdos desde las potencialidades, las afirmaciones y la palabra generativa.

Finalmente, el potencial reflexivo se da en la medida en que el niño o la niña desarrolle su capacidad de darse cuenta de sí mismo y del lugar que ocupa en la relación que establece con los otros y con el mundo, permitiendo reflexionar sobre dinámicas de respeto, reconocimiento, solidaridad, sentido de lo justo, equitativo y la ampliación del círculo ético.

Rodríguez, citando a Nussbaum respecto al papel que desempeñan las emociones en el razonamiento práctico, afirma que son tan necesarias como la creación de las capacidades
Considera que los elementos cognitivos que están presentes en la emoción permiten a las personas modelar su contenido y también abrirlas a la influencia de las construcciones sociales. De este modo, la perspectiva cognitivo-evaluadora de la emoción apunta la posibilidad de que la emoción misma pueda ser evaluada y alterada en caso de no superar la crítica efectuada por un examen racional. (Nussbaum como se citó en Rodríguez, 2012, p. 168)

Desde el enfoque de las capacidades como una propuesta para el desarrollo humano, Nussbaum desarrolla el concepto de capacidades retomado en la obra de Gough (2007) como:
Lo que las personas son realmente capaces de hacer y de ser. Su obra está muy vinculada con la de Amartya Sen, para quien los funcionamientos "constituyen partes del estado de una persona" y, teniendo en cuenta que dichos funcionamientos son "intrínsecamente valiosos", representan estados de bienestar. Las capacidades se refieren al conjunto de funcionamientos que son factibles para una persona, pudiendo elegir. (p. 178)

En este sentido, resulta primordial cultivar las capacidades en los niños y las niñas desde sus primeros años de vida, a través de una educación para la paz basada en la lúdica, el juego, el arte y la literatura, que permita al niño o niña percibir e imaginar al otro en el desarrollo de prácticas de empatía como la compasión, reciprocidad, cuidado y afecto desde la red parental. Según la Ley 1098 del 2006, por la cual se expide el Código de Infancia y Adolescencia de Colombia se define la parentalidad como: 
Investigación para la paz: perspectiva de paz desde la primera infancia y sus agentes relacionales

\begin{abstract}
Una de las funciones básicas de la familia y la dotación de los niños y niñas mediante procesos formativos para vivir una vida digna y con calidad, así como la protección contra toda forma de violencia y discriminación, al reconocerlos como Niños y Niñas sujetos de derecho. (Ley 1098 de 2006)
\end{abstract}

En la misma línea, Rodrigo, Martín, Cabrera y Máiquez (2009) definen las competencias parentales como:

El conjunto de capacidades que permiten a los padres afrontar de forma flexible y adaptativa la tarea vital de ser padres, de acuerdo con las necesidades evolutivas y educativas de los hijos/as y con los estándares considerados como aceptables por la sociedad, y aprovechando todas las oportunidades y apoyos que les ofrecen los sistemas de influencia de la familia para desarrollar estas capacidades. (p. 115)

De acuerdo con lo anterior, esta investigación social tuvo la apuesta por la construcción de un entramado de sentidos y significados alrededor de la cultura del buen trato en la que se reconoció a los niños y las niñas como otredades y alteridades capaces de pensar, sentir, decidir, actuar con otros y para otros, capaces de comprender y lenguajear el mundo externo a partir de consensos entre el mundo de sus pensamientos y las prácticas parentales; esto requirió la reconfiguración no solo del concepto teórico de las competencias parentales sino también de los imaginarios culturalmente aprendidos e incorporados en las vivencias del entorno familiar.

Por esta razón, el desarrollo de los potenciales del desarrollo humano es transversal a las vivencias cotidianas, reconociendo las prácticas de crianza, reflexionando sobre estas y entendiendo su acción/comunicación con el fin de configurar escenarios de crianza para la paz desde la primera infancia ${ }^{2}$ en los centros de desarrollo infantil (modalidad institucional) que en adelante se abreviara con las siglas CDI, del municipio de Marmato ${ }^{3}$, ubicado al noroeste del departamento de Caldas.

\footnotetext{
${ }^{2}$ Se entiende por primera infancia el periodo de la vida, de crecimiento y desarrollo comprendido desde la gestación hasta los siete años aproximadamente y que se caracteriza por la rapidez de los cambios que ocurren. Esta primera etapa es decisiva en el desarrollo, pues de ella va a depender toda la evolución posterior del niño en las dimensiones motora, lenguaje, cognitiva y socioafectiva, entre otras.

${ }^{3}$ Marmato es un municipio ubicado al noroeste de Caldas, famoso por sus minas de oro. Recorrido en el año de 1536 por Sebastián de Belalcázar y en 1537 por Juan Badillo, su nombre proviene de una piedra en forma de hexaedro llamada pirita o marmaja. Es el cuarto municipio más viejo de Colombia.

Por su ubicación desafiante loma arriba sobre la arisca ladera del cerro El Guamo, Marmato fue bautizado hace muchos años con el apelativo de "Pesebre de oro de Colombia". Históricamente, Marmato ha sustentado su economía en la explotación minera, constituyéndose esta actividad en la principal fuente de ingresos y empleo para sus habitantes.
} 


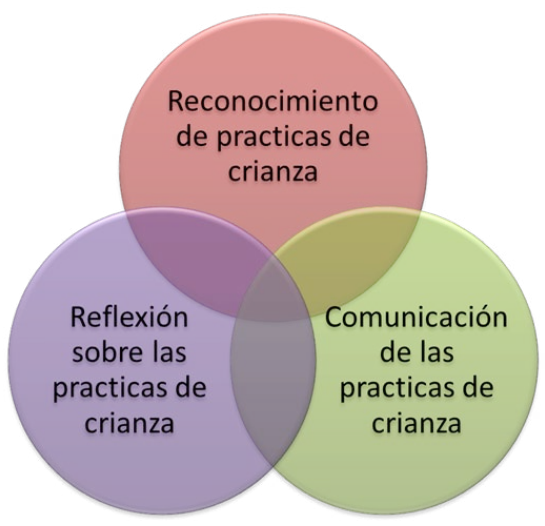

Grafica 1. Apuesta formativa.

Fuente: Apuesta formativa del proyecto de investigación.

\title{
Metodología
}

Esta investigación social de corte cualitativa tuvo una duración de dos años en el marco de la Maestría en Educación y Desarrollo Humano del CINDE y la Universidad de Manizales, entre los años 2016-2017.

Delimitación de actores y de espacio: la unidad de trabajo se conformó por 22 agentes educativas de la primera infancia y 10 madres de familia usuarias de la estrategia de Cero a Siempre del CDI Goticas de Oro en tres sedes ubicadas en el barrio el Llano, San Juan y Montecarlo.

El paradigma socio-crítico o crítico social ${ }^{4}$ orientó el análisis de la investigación, permitiendo reconocer la realidad como una praxis social orientada hacia la emancipación y liberación.

Según Popkewitz (como se citó en Alvarado y García, 2008)

\begin{abstract}
Algunos de los principios del paradigma socio crítico son: (a) conocer y comprender la realidad como praxis; (b) unir teoría y práctica, integrando conocimiento, acción y valores; (c) orientar el conocimiento hacia la emancipación y liberación del ser humano; y (d) proponer la integración de todos los participantes, incluyendo al investigador, en
\end{abstract}

\footnotetext{
${ }^{4}$ Desde el ámbito de la investigación, un paradigma es un cuerpo de creencias, presupuestos, reglas y procedimientos que definen cómo hay que hacer ciencia; son los modelos de acción para la búsqueda del conocimiento. Los paradigmas, de hecho, se convierten en patrones, modelos o reglas a seguir por los investigadores de un campo de acción determinado (Alvarado y García, 2008).

De acuerdo con Arnal citado por Boza "el paradigma socio-crítico adopta la idea de que la teoría crítica es una ciencia social que no es puramente empírica ni sólo interpretativa; sus contribuciones, se originan, "de los estudios comunitarios y de la investigación participante (...)Tiene como objetivo promover las transformaciones sociales, dando respuestas a problemas específicos presentes en el seno de las comunidades, pero con la participación de sus miembros” (Boza, 2011).
} 
Investigación para la paz: perspectiva de paz desde la primera infancia y sus agentes relacionales

procesos de autorreflexión y de toma de decisiones consensuadas, las cuales se asumen de manera corresponsable. (p. 190)

Desde esta perspectiva se asume a los niños, las niñas, sus familias y agentes educativas como actores sociales que aportan a la construcción de paz, a partir de la interacción con otros desde acciones cotidianas y comunitarias manifestadas en las dinámicas culturales y sociales.

La identificación de estas prácticas permite reconocer y comprender el contexto de Marmato como un lugar pluriétnico y diverso que a través de la praxis parte de la experiencia vivida y construida en escenarios propicios para la intervención/acción orientada a la crianza para la paz.

A partir de la integración de los tres actores, se generó un diálogo intergeneracional que permitió aflorar los conocimientos y las prácticas que las agentes educativas y madres de familia constituían desde sus historias de vida, por esta razón, y como una estrategia de mediación de este enfoque, la autorreflexión llevó a cada una asumir una toma conciencia sobre la importancia de transformar algunos patrones y prácticas de crianza que habían naturalizado en las competencias parentales; ello permitió orientar la metodología hacia la emancipación y la toma de decisiones consensuadas que asumieron en una corresponsabilidad compartida desde la institucionalidad, la familia y la comunidad.

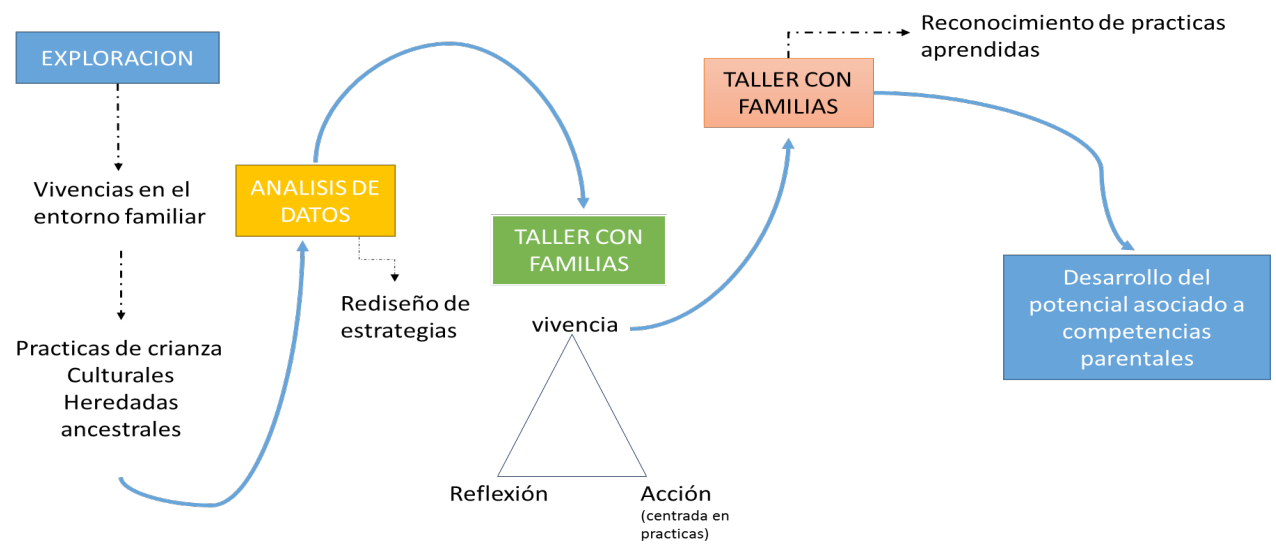

Grafica 2. Estrategia Metodológica para el Agenciamiento Social.

Fuente: Estrategia Metodológica del proyecto de investigación. 


\section{Diseño metodológico}

Se plantearon cuatro fases que orientaron el proceso investigativo de manera flexible y participativa.

La primera fase preliminar permitió la delimitación del tema interés investigativo, los objetivos, el acercamiento al contexto del municipio, la negociación y acuerdos para la ejecución del proyecto de investigación con el Instituto Colombiano de Bienestar Familiar (ICBF).

La segunda fase, diagnóstico-participativo, tuvo como objetivo reconocer las interacciones sociales del contexto, reconociendo la idiosincrasia, las creencias, la cosmovisión, las percepciones que se tiene de los niños y las niñas de la primera infancia; a partir de la técnica de cartografía social y comunitaria que facilitó la identificación de las competencias parentales de los agentes relaciones.

De acuerdo con la teoría ecológica de Bronfenbrenner es necesario considerar el entorno ecológico para entender el desarrollo humano, según este modelo,

Elambiente ecológico estáintegrado por cincosistemasinterrelacionados:

(a) el microsistema, que hace referencia a las interacciones, roles sociales y relaciones interpersonales que se experimentan durante el desarrollo en el entorno inmediato (i.e., la familia, el barrio y la escuela); (b) el mesosistema, que incluye la relación entre dos o más de los microsistemas (e. g., la relación entre el hogar y la escuela); (c) el exosistema, que comprende los procesos y relaciones que influyen indirectamente en el desarrollo del niño o niña, al interactuar con los factores de su microsistema; (d) el macrosistema, que hace referencia a la cultura y la subcultura de la sociedad; y (e) el cronosistema, que hace referencia a los cambios cronológicos personales y ambientales que pueden influir en el desarrollo. (Alvarado y García, 2008, p. 190)

La tercera fase, praxis transformadora, fue transversal al proceso investigación/acción al poner en juego talleres pedagógicos, lúdicos y creativos en los cuales participaban niños, niñas y sus agentes socializadores, enfocados en el fortalecimiento de las prácticas de crianza orientadas al buen trato, la capacidad de agenciamiento de los potenciales humanos, en la habilidad para jugar explorando el mundo de las emociones y los sentimientos que pasan por el potencial del cuerpo y el potencial reflexivo en la configuración de la crianza para la paz.

Laformación de sujetos que sean capaces de tomar decisiones responsables frente a las consecuencias de sus actos sobre el medio ambiente y que se conviertan en agentes de cambio para sus comunidades. Para ello 
Investigación para la paz: perspectiva de paz desde la primera infancia y sus agentes relacionales

\begin{abstract}
se agencia la creación de espacios de intercambio, problematización y formación en los cuales se creen puentes entre los niños, las niñas, los jóvenes y las jóvenes, comunidades e instituciones. (Salgado, Patiño y Ospina, 2011, p. 118)
\end{abstract}

La cuarta fase, trascendiendo la huella, se ejecutó a través de la estrategia de multiplicación, en la que se compartían y socializaban los aprendizajes construidos conjuntamente en la experiencia, presentados en diferentes escenarios comunitarios, con el fin de empoderar y transformar otros lugares habitados por los niños y las niñas.

Según Habermas (como se citó en Alvarado y García, 2008):

El saber es el resultado de la actividad del ser humano motivada por necesidades naturales e intereses. Se constituye desde tres intereses de saberes llamados por él como técnico, práctico y emancipatorio. Cada uno de esos intereses constitutivos de saberes asume forma en un modo particular de organización social o medio, y el saber que cada interés general da lugar a ciencias diferentes. (p. 193)

El proceso de codificación, análisis, interpretación y construcción de la experiencia se hizo en el Software ATLAS.ti como un ejercicio inductivo, no sólo identificando las competencias parentales sino también comprendiendo el acontecimiento en los relatos de las prácticas de crianza.

\title{
Diplomado crianza para la paz
}

La estructura metodológica que se diseñó a partir de lo logrado en el Diplomado crianza para la paz tuvo como base la propuesta educativa "Niños, Niñas y Jóvenes Constructores de Paz" y "Convidarte para Paz" del Centro de Estudios Avanzados en Niñez y Juventud del CINDE y la Universidad de Manizales, a partir de los tres momentos pedagógicos que propone: 1) formación, encuentros de aprendizaje recíproco, 2) multiplicación, escenarios de socialización y expansión de la experiencia formativa e investigativa con sus pares, 3) jornadas por la niñez, acciones colectivas en el contexto Institucional CDI y familiar.

El diplomado "Crianza para la Paz” se realizó a través de diez talleres lúdico- creativos que incluyeron técnicas para la recolección de información, cartografía social, narrativas colectivas, historias de vida y observación participante.

Abordar la primera infancia desde la perspectiva sistémica permite la interacción entre la familia, la institucionalidad y la sociedad civil como actores en la construcción de paz, fortaleciendo las habilidades pro-sociales a partir de procesos asociativos directos entre 
experiencias ajenas y experiencias pasadas y estimulando la capacidad cognitiva de asumir roles empáticos y altruistas. Son entendidas como:

Aquellos comportamientos que, sin la búsqueda de recompensas externas, extrínsecas o materiales, favorecen a otras personas, grupos o metas sociales y aumentan la probabilidad de generar una reciprocidad positiva, de calidad y solidaridad en las relaciones interpersonales o sociales consecuentes, salvaguardando la identidad, creatividad e iniciativa de las personas o grupos implicados. (Olivar, 2004, p. 39)

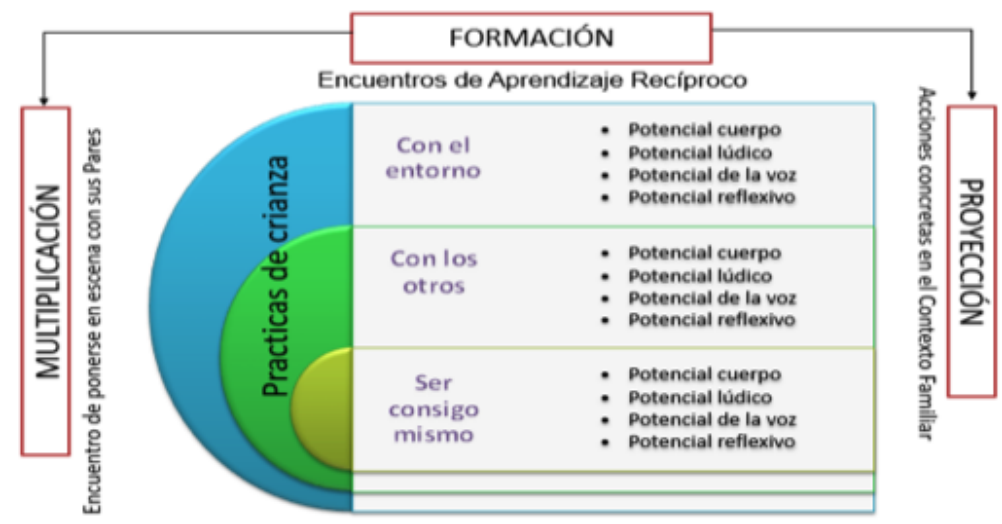

\section{Estrategia Metodológica}

\section{Grafica 3. Potenciales del desarrollo humano de los niños y las niñas de la primera infancia. \\ Fuente: Programa Niños, niñas y Jóvenes Constructores de Paz y Convidarte para la paz.}

\section{Resultados}

Desde la teoría sociológica de Pierre Bourdieu esquematizar los espacios simbólicos y los lugares físicos donde los niños y las niñas aprenden hábitos y adquieren capitales, es importante, porque es allí donde participan como sujetos sociales y donde se internaliza y se reproducen las relaciones de poder; en este sentido se analizarán tres escenarios de socialización: la familia como institución, el CDI y la comunidad. 
Investigación para la paz: perspectiva de paz desde la primera infancia y sus agentes relacionales

La familia como primer escenario de socialización y de análisis establece una jerarquía que, de acuerdo al campo, es modificada por las relaciones de fuerza y de poder mediadas entre los diferentes actores del sistema familiar (padre, madre otros agentes socializadores e hijos). Bourdieu en Guerra (2010) precisa que: "el capital cultural objetivado no existe y no subsiste como capital cultural material y simbólicamente actuante más que en, y por las luchas que se desarrollan, el terreno de los campos de producción cultural” (p. 399).

El tipo de familia de América Latina por lo general es la que trajeron los españoles y portugueses, se funda en bases judío-cristianas de estirpe patriarcal que giran la autoridad alrededor del padre. Sin embargo, "el concepto de familia es dinámico y está en constante evolución. La definición legal de este término va a depender de la legislación de cada estado o país y, generalmente, se encuentra ubicada en la constitución” (Gómez y Villa, 2014, p. 17).

En Colombia, la familia constituida por vínculos naturales o jurídicos es reconocida por la Ley 294 de 1996 y enumera como sus integrantes a:

Cónyuges o compañeros permanentes, el padre y la madre aunque no convivan bajo un mismo techo, los ascendientes o descendientes de los anteriores, los hijos adoptivos y todas las personas que de manera permanente se hallen integrados a la unidad doméstica. (Ley 294 de 1996)

Sin embargo, teniendo en cuenta las dinámicas y estructuras familiares de quienes participaron en el estudio, se amplía el concepto de familia retomando la Ley 82 de 1993 que reconoce a la "mujer cabeza de familia"

A quien siendo soltera o casada, tenga bajo su cargo, económica o socialmente, en forma permanente, hijos menores propios u otras personas incapaces o incapacitadas para trabajar, ya sea por ausencia permanente o incapacidad física, sensorial, síquica o moral del cónyuge o compañero permanente o deficiencia sustancial de ayuda de los demás miembros del núcleo familiar. (Ley 82 de 1993)

La caracterización de las familias participantes se compone de distintas tipologías: 3 de las 8 familias están compuestas por la figura del padre, madre e hijos, es decir, configuran una familia nuclear; 2 de las 8 familias son extensas conviviendo tíos, abuelos, niños, niñas y 3 de ellas son monoparentales, en donde la mujer es cabeza de familia.

La principal fuente de ingresos de estas familias es la extracción de minerales, arte tradicional del municipio, sin embargo, este fenómeno oculta una problemática que fue identificada a partir de observación participante y la entrevista semi-estructurada con líderes comunitarios, señalando el trabajo infantil como una actividad cotidiana que viven los niños y las niñas. 
Este oficio es percibido por los adultos como una actividad sin mayores riesgos y como una oportunidad y colaboración al ingreso económico. Desde el estudio de la familia, algunos autores han reflexionado sobre los roles que se tiene en las familias considerando

\begin{abstract}
Desde su origen, la familia tiene varias funciones que podríamos llamar universales, tales como: reproducción, protección, la posibilidad de socializar, control social, determinación del estatus para el niño y canalización de afectos, entre otras. La forma de desempeñar estas funciones variará de acuerdo a la sociedad en la cual se encuentre el grupo familiar . (Gomez y Villa, 2014, p. 12)
\end{abstract}

En la identificación de las competencias parentales se reconoció que los niños y las niñas de la primera infancia no son interlocutores válidos para la toma de decisiones de los adultos, prevaleciendo una mirada adulto-céntrica que silencia las voces de la infancia. Por esta razón, la corresponsabilidad entre las familias y las agentes educativas debe constituirse en el punto de partida para la construcción del lenguaje semiótico, simbólico y cultural, creando escenarios en donde los niños y las niñas sean reconocidos como sujetos pensantes, racionales, emocionales y, por tanto, capaces de ponerse ante otros en la construcción de su propia realidad.

Frente a este diagnóstico, la investigación acción implementa una estrategia formativa y pedagógica que permitió aportar y fortalecer las competencias parentales en las redes familiares, al reflexionar y comunicar alternativas a las tradicionales que potenciaron nuevas formas de relacionamiento entre los niños, las niñas y sus cuidadores.

Así mismo, contribuyó en las escuelas de padres que fueron lideradas por las ocho madres de familia; el rol de multiplicadores se adopta, con el propósito de enunciar otras prácticas de crianza para la paz desde las voces y experiencias vividas por este grupo focal. Esto empoderó a las participantes directas y fue la oportunidad para que otros cuidadores reconocieran sus propias pautas de crianza y reflexionaran en torno a las herramientas desplegadas por el diplomado.

La actividad que más me gustó fue la multiplicación, porque allí se veían padres comprometidos con las necesidades de sus hijos, donde como padres volvimos hacer niños participando de cada actividad. (Narrativa madre de familia, comunicación personal, 8 de octubre de 2016)

El segundo escenario, el CDI, entendido como un campo de luchas de estructuras sociales y simbólicas, ya que legitima ciertos discursos y prácticas en la concepción de la primera infancia desde la mirada de la institucionalidad de ICBF, donde se ponen en juego los recursos y dispositivos para el reforzamiento de la socialización y por ende de legitimización de los diferentes roles. 
Investigación para la paz: perspectiva de paz desde la primera infancia y sus agentes relacionales

\begin{abstract}
Permitir que hagan ciertas cosas, con supervisión de un adulto. Que tomen decisiones con autonomía, pero con responsabilidad de nosotros, para vigilarlos. Por una exigencia de nuestro trabajo. Es que no es lo mismo que con los hijos de nosotras porque esos son de uno, estos son como hijos, pero prestados y las mamitas son muy cansonas por eso nos joden, uno mantiene con miedo de que les pase algo porque el ICBF se le va encima a uno, es mejor que los niños no hagan muchas cosas afuera. (Narrativa agente educativa, comunicación personal, $3 \mathrm{de}$ noviembre de 2016)
\end{abstract}

El agente educativo no es por tanto autor de sus prácticas educativas, en el actúa invisiblemente la fuerza social, generando límites y temores para ser autónomas, Bollinger (1991), citando a Bourdieu explica en la entrevista para el Centro Nacional de Documentación Pedagógica (CNDP) “...el sistema escolar, en la sociología moderna, es uno de los lugares donde se fabrican las personas, donde se crean las formas de pensar, las formas de actuar, en relación directa con la familia” (p. 1).

Lo anterior se evidenció al acompañar el proceso educativo de las docentes, identificando un desafío en la educación inicial en la que los niños y las niñas inician la exploración de la vida en instituciones contemporáneas y obsoletas a la vez que les quita la magia, el espacio y la libertad de desplegar la potencialidad humana del juego, la lúdica, el cuerpo, la creatividad, la curiosidad, la capacidad de asombro y el aprendizaje experiencial.

En el tercer escenario, la comunidad, aparece la construcción de las relaciones de amistad establecidas con sus pares, en los cuales los niños y las niñas interactúan a partir de consensos y negociaciones compartidas, visibilizadas en el juego de roles, reinventando nuevas formas de relacionarse.

Aparece una línea de fuga entre los campos de la familia, la institucionalidad y el parque comunitario, ya que se genera una ruptura entre las fuerzas de poder, las relaciones de dominación, los discursos hegemónicos y los dispositivos técnicos, y emerge la subjetividad política de los niños y las niñas al aparecer en el espacio público, configurando un entramado de relaciones próximas, basadas en la autonomía, confianza, afecto, amistad, solidaridad y la cooperación, “(...) jugar con los amiguitos, dándonos abrazos, picos, en el parque en el columpio ayudamos a mi hermanita" (María Alejandra Álvarez, comunicación personal, 18 de agosto de 2016)

Según Zuleta (como se citó en Gallego, 2015) la democracia implica la “aceptación de la angustia de tener que decidir por sí mismo" (p.157). En otras palabras, es la capacidad de autonomía que ha de asumir cada persona frente a las situaciones que la vida le presenta.

${ }^{5}$ nombre real de una niña de la primera infancia, participante del proyecto de investigación 
Al momento de analizar la categoría de participación infantil y democracia en la primera infancia, emergieron expresiones de las madres y las agentes educativas afirmando lo siguiente:

Es demasiado pequeño para decidir; él debe obedecer; yo pongo el horario para ver los muñecos porque por él se queda ahí pegado; yo le ayudo a escoger la ropa porque ella no sabe si le combina; los niños son manipuladores, ellos hacen berrinche y creen que uno va hacer lo que ellos quieren. (Narrativas madres de familia, comunicación personal, 19 de octubre de 2016)

Estas formas de autoritarismo desencadenan actitudes de obediencia y relaciones de poder desde una perspectiva adulto-céntrica; "esta situación evidencia el poco o nulo reconocimiento que se tenía de la infancia y de sus características particulares, así como la poca importancia que se le daba a la participación infantil” (Gallego, 2015, p. 154).

La propuesta educativa para el agenciamiento social “Crianza para la paz” desplegó herramientas prácticas a partir de actividades y juegos lúdicos-pedagógicos que buscaban fortalecer la autonomía en los niños y las niñas, en los cuales el rol de los agentes socializadores era propiciarles un ambiente de confianza, estimulación y reconocimiento de sus capacidades creativas para relacionarse con el otro. Este escenario de encuentro entre adultos y niños potenció las dimensiones del desarrollo humano como ejes transversales en la construcción de paz desde los primeros años de vida y permitió pasar de la heteronomía a la autonomía.

Para Zaragoza (2009) la cultura de paz:

Es un conjunto de valores, actitudes y comportamientos que reflejan el respeto a la vida, al ser humano y su dignidad. La cultura de paz pone en primer plano los derechos humanos, el rechazo a la violencia en todas sus formas y la adhesión a los principios de libertad, justicia, solidaridad y tolerancia, así como la comprensión entre los pueblos, los colectivos y las personas. (Muñoz, 2009, p. 2)

Los niños y niñas son constructores de paz a partir de acciones cotidianas que expanden su capacidad creativa e imaginativa para desplegar su potencial afectivo, de cuidado y cooperación, su capacidad de reconciliación y por tanto de mediación en los conflictos, poniendo en juego sus múltiples lenguajes corpóreos, lúdicos y de exploración y agenciando desde la autonomía una cultura de paz donde converge la solidaridad como máxima expresión.

El rol de las agentes educativas en pro de una cultura de paz se hace fundamental a través de las estrategias pedagógicas y didácticas que construyen en sus planeadores. 
Investigación para la paz: perspectiva de paz desde la primera infancia y sus agentes relacionales

La orientación que se hizo en los grupos de estudio y trabajo (GET) fortaleció las propuestas alternativas de intervención con los cuidadores desde una apuesta intergeneracional, en las cuales como agentes socializadores se involucren activamente en la cultura del buen trato y por ende sujetos constructores de paz, quienes a su vez hacen el rol de multiplicar las herramientas pedagógicas en su contexto familiar y comunitario.

La cultura de paz necesita sujetos que sean reconocidos como constructores capaces de actuar desde y para la paz, no solo en su círculo más próximo, sino imaginando y trascendiendo su capacidad de ocuparse por los otros desde una práctica de alteridad. Ello cobra sentido en la medida en que los cuidadores van incorporando elementos fundamentales para una crianza positiva que parte de la escucha y el reconocimiento legítimo de las voces de los niños y las niñas, el acompañamiento de las agentes educativas en el fortalecimiento de las competencias parentales y en la formación de habilidades prosociales desde los primeros años.

Las próximas investigaciones relacionadas con la cultura de paz en la primera infancia, debe partir de la identificación de las competencias parentales como aquellas prácticas que se van interiorizando y reproduciendo en la vida cotidiana sin ser reflexionadas, confrontadas, interpeladas, y cuestionadas, y que por tanto son transmitidas de generación en generación.

Movilizar otras perspectivas desde la mirada apreciativa de los niños y las niñas de potenciar en ellos maneras de ser y estar desde la posibilidad y oportunidad de creación en el encuentro con el otro, siendo desde su esencia niños y niñas, curiosos, sensibles, espontáneos, extrovertidos, y capaces de resignificar las prácticas de crianza de sus familias desde los potenciales del desarrollo humano.

La experiencia que viví fue muy significativa y enriquecedora al compartir y trabajar con los padres, todos unidos en pro del bienestar de todos los niños de nuestro hermoso municipio, el día que más recuerdo es el día que trabajamos la multiplicación de la ética y el cuidado de la crianza. (Narrativa madre de familia, comunicación personal, 3 de noviembre de 2016)

El reconocimiento de los actores permitió leerse como cuidadores capaces de aprender y enseñar paralelamente; es decir, tanto madres como docentes intercambiaron experiencias previas sobre la crianza y la cultura de paz en un dialogo de saberes, emergieron 'consejos' y discusiones sobre los comportamientos de los niños y las niñas y los desafíos que tiene la cultura del buen trato.

Lo que más me impactó o gustó del diplomado fue el poder compartir con algunas madres de familia, con las cuales se pudo compartir conocimientos sobre las pautas de crianza llevadas dentro del hogar 
a prácticas dentro del CDI me servirá para mi futuro, para poner en práctica con mi hija, mi comunidad y mi entorno. (Narrativa madre de familia, comunicación personal, 3 de noviembre de 2016)

Finalmente, el compromiso ético político es trascender las fronteras del conocimiento y llegar a los lenguajes orales, corporales, semánticos y culturales de las personas con quienes abordamos estos fenómenos sociales. En coherencia con el paradigma socio-crítico o crítico social se recoge esta experiencia en un material didáctico "Cartilla Crianza para la Paz" financiada por Colciencias, para facilitar a los agentes socializadores herramientas pertinentes que aporten al fortalecimiento de los potenciales de los niños y las niñas reconocidos como sujetos legítimos y válidos en la construcción de paz desde sus primeros años.

Por lo tanto, quedan preguntas que permitirán profundizar o vislumbrar nuevas investigaciones focalizadas en la primera infancia, sus familias y otros agentes socializadores: ¿Qué lugar ocupan los abuelos y otros cuidadores como tíos, tías, vecinos y redes de apoyo en una crianza para la paz? ¿Cómo generar procesos de participación en las escuelas de padres que sean lideradas desde ellos y para ellos? ¿Cómo medir el impacto y la sostenibilidad de proyectos alternativos de intervención con los cuidadores? ¿Cuál es el papel de los padres de familia en la crianza para la paz? ¿Cómo democratizar las funciones y roles de los cuidadores? ¿Cómo configurar sujetos políticos desde la primera infancia, capaces de pensar y actuar en el entorno que los rodea como agentes constructores de paz? ¿Cómo co-construir escenarios de crianza y socialización de niños y niñas constructores de paz?

\section{Discusión}

\section{Una mirada a la infancia desde la perspectiva de paz}

La familia es el primer contexto de desarrollo, el primer ámbito en el que
el niño modelará su construcción como persona, en el que establecerá
las primeras relaciones con otros y en el que creará una imagen de sí
mismo y del mundo que le envuelve. Dicho desarrollo se producirá en
un escenario de relaciones, intenciones y actuaciones en las que el niño
desempeñará un papel protagonista y activo. La calidad y cualidad de las
relaciones interpersonales que viva en este contexto dejarán una huella
significativa en su esencia como ser individual y social. (Guasch i Garcia
y Domínguez, 2012, p. 115)

La infancia será una generación capaz de mirar con otros ojos la barbarie de la guerra, las heridas del paisaje, la intemperie de la violencia y empezará a afirmar la vida con los otros en prácticas cotidianas de re-existencia en medio de un hecho social como el post acuerdo; para ello, los agentes educativos, cuidadores, sociedad civil, los agentes de política pública, las instituciones 
Investigación para la paz: perspectiva de paz desde la primera infancia y sus agentes relacionales

que garantizan el bienestar de esta población, necesitan preparar y reparar a los niños y las niñas para la paz, es decir, transformar sus lenguajes, imaginarios, percepciones y acciones que se han naturalizado a lo largo de la historia de 52 años de conflicto interno en el país.

La resignificación de los territorios y lugares simbólicos que habitan los niños y niñas configuran un tejido social que parte de las potencialidades, habilidades y destrezas manifestadas en sus dimensiones corporales, emocionales y cognitivas, para una reinvención de la mirada desde el afecto, la ética y la compasión como sujeto político que ocupa y aporta al mundo de la infancia a través del juego, la lúdica, el baile, la espontaneidad y la capacidad de exploración de lo que los rodea.

Sin embargo, se hace necesario que los niños y las niñas se acerquen a la historia de Colombia desde la memoria colectiva para garantizar la no repetición de hechos atroces que vulneran la raza humana, y que sea este un camino a recorrer con el pasado al frente, para no olvidar y aprender cada generación de las huellas profundas que ha dejado el conflicto armado con el reclutamiento de menores de edad en las filas armadas.

Por tanto, una educación para la paz y el buen trato instaura en los agentes socializadores un ambiente de empatía, comprensión, comunicación asertiva y afectiva en el acompañamiento de los niños y niñas. Estas prácticas permiten reconocerlos como sujetos con habilidades y capacidades prosociales vinculadas a las interacciones más cercanas con sus cuidadores y pares. Eisenberg citado en Empatía y altruismo en los procesos de alteridad de niños de educación primaria por Hernández (2012) define la conducta prosocial como:

Aquel comportamiento voluntario que pretende beneficiar a otro, por ejemplo, las conductas que tienen como objetivo ayudar, compartir y confrontar. Los psicólogos consideran que muchos comportamientos prosociales están motivados por factores como la esperanza de recompensas concretas, la aprobación social o el deseo de aliviar los propios estados internos negativos (por ejemplo, la culpa o la tristeza que produce observar a una persona necesitada). Pero las conductas prosociales motivadas por la empatía. (p. 156)

En esta experiencia, las habilidades prosociales de los niños y niñas se relacionan con el contexto social en el que viven y de los cuales provienen sus familias. Entornos de alto índice de violencia intrafamiliar y, por consiguiente, los niños y niñas adoptan e incorporan prácticas violentas referidas por sus cuidadores, así lo afirma Martínez (2009), citando a Shapiro: "Los niños están afectados por la falta de atención familiar y su desarrollo emocional es mucho más vulnerable ante situaciones estresantes por la falta de modelos adecuados para un desarrollo emocional" (p. 115). 


\section{Cultura del buen trato}

Según Barudy (2005), el concepto del buen trato se basa en,

La capacidad de tratar bien a las crías como capacidad inherente a las posibilidades biológicas de los seres humanos. Nuestra estructura biológica permite el carácter social y altruista de nuestros comportamientos por las hormonas que produce, entre otras: la oxitocina, la vasopresina y las endorfinas. Cuidar de los niños y niñas ofreciéndoles contextos de buenos tratos es un fenómeno posible y al alcance de cualquier comunidad humana. (p. 1)

En otras palabras, pone en el centro el rol de los adultos al proveer el bienestar y la salud de todos los niños, las niñas y adolescentes

Es responsabilidad de cada adulto, cada comunidad y cada Estado, crear las condiciones para que todos los niños y niñas tengan acceso a los cuidados, la protección y la educación que necesitan para llegar a la adolescencia y luego integrar la vida adulta de una forma sana, constructiva y feliz. (Barudy, 2005, p. 1)

De acuerdo con lo anterior, los agentes socializadores son una garantía para que los niños y niñas configuren nuevas ciudadanías desde una postura ética y, por ende, desplieguen sus capacidades políticas, mediadas en la autonomía y la participación como prácticas necesarias para establecer relaciones consigo mismo, con los otros y con el entorno.

Según el Centro Internacional de Educación y Desarrollo Humano (2004), "el buen trato es una modalidad de convivencia cotidiana que genera una transformación cultural a partir de la construcción colectiva de vínculos sanos” (p. 5). Es decir, es una forma de relacionamiento que se basa en el potencial del afecto, entendido como una dimensión que fortalece la autoestima y el reconocimiento del otro, a partir de entramados de sentidos y significados que permiten intersubjetivamente maneras-otras de interacción.

Por tanto, la cultura del buen trato requiere fortalecer las competencias parentales, que para esta experiencia particular se hizo a partir de la promoción de actitudes positivas que generaron prácticas de crianza de afecto, y cuidado, en las que los niños y niñas participaron desde sus capacidades y habilidades en la transformación creativa de los conflictos.

La parentalidad resiliente es la que ejerce la madre o el padre como apoyo afectivo a sus hijos en los sucesos de la vida, que son parte de un proceso continuo donde existen perturbaciones y consolidaciones. En 
Investigación para la paz: perspectiva de paz desde la primera infancia y sus agentes relacionales

el desafío de existir, las dificultades pueden ser fuente de crecimiento siempre y cuando el niño encuentre en una madre o en un padre el apoyo necesario para enfrentarlas y darles un sentido. (Barudy y Dantagnan, 2005, p. 46)

\section{Cultura de paz}

El concepto cultura de paz es emitido por primera vez en la Asamblea General de las Naciones Unidas, estableciendo que es

[...] conjunto de valores, actitudes, y conductas que plasman y suscitan

a la vez interacciones e intercambios sociales basados en principios de libertad, justicia y democracia, todos los derechos humanos, la tolerancia y la solidaridad; que rechazan la violencia y procuran prevenir los conflictos, tratando de atacar sus causas y buscando soluciones a los problemas mediante el diálogo y la negociación, y que garantizan el pleno ejercicio de todos los derechos y proporcionar los medios para participar plenamente en el proceso de desarrollo de su sociedad. (Fisas, 2011, p. 3)

En este sentido, la cultura de paz desde la primera infancia tiene como punto de partida la empatía, que forja con los otros el respeto por la diferencia y valora el potencial creativo de la pluralidad. Según Hans Kung (como se citó en Fisas, 2011): "Educar para la paz significa preparar a las nuevas generaciones para buscar un nuevo consenso fundamental sobre convicciones humanas integradoras" (p. 3).

La cultura de paz es una construcción social que no se debe agotar en el ámbito educativo sino que involucra las familias, los niños y las niñas, mediante un proceso de transformación de la naturalización de las violencias a prácticas de alteridad, "este nuevo enfoque es el que persigue la "cultura de paz”, o "cultura para la paz", si la entendemos como un proceso que, en primera instancia, habrá de transformar la "cultura de la violencia”, tan presente en nuestras sociedades" (Fisas, 2011, p. 3).

Así mismo, Galtung (como se citó en Concha, 2009) afirma en la teoría de los conflictos la necesidad de hacer una regulación positiva de los conflictos, convirtiendo las situaciones conflictivas en experiencias pedagógicas, de concientización, de empoderamiento, de estímulo y desarrollo de la creatividad.

El desafío de la cultura de paz desde la primera infancia pasa por el rechazo de aquellas conductas sociales que naturalizan el uso de la fuerza y la violencia como formas de relacionamiento entre los adultos, los niños y niñas y la cultura del buen trato reivindica las prácticas y experiencias 
vividas desde el afecto, el respeto, el reconocimiento, la reconciliación y la protección.

La cultura de paz es una tarea educativa que pasa por educar en y para el conflicto, en desenmascarar la violencia cultural y el patriarcado, en educar para la disidencia, el inconformismo y el desarme, en responsabilizarnos, en movilizarnos, en transformar los conflictos, en llevar a cabo el desarme cultural, en promover una ética global y en buscar un consenso fundamental sobre convicciones humanas integradoras, entre otras cosas. (Fisas, 2011, p. 3)

\section{Conclusiones}

1. La cultura de paz desde la primera infancia se fortalece en el despliegue de los potenciales del desarrollo humano como una pedagogía orientada al agenciamiento, que reconoce a los niños y las niñas como sujetos políticos capaces de pensar y actuar desde el afecto, el cuidado de sí mismo, del otro y lo otro, la capacidad creativa para la transformación y mediación de los conflictos familiares y comunitarios, el desarrollo de sus múltiples lenguajes y expresiones comunicativas y prioriza el juego como herramienta lúdica y estética que fortalece el relacionamiento y la interacción entre ellos con sus pares y los adultos.

2. El ICBF plantea en sus lineamientos la formación y el acompañamiento a familias como parte integral de las acciones de atención de la modalidad institucional de educación inicial, por tanto, fortalecer en el marco de la investigación la escuela de padres permitió rediseñar estrategias que fortaleció los planes operativos de atención integral (POAI) del CDI Gotitas de Oro. Se buscaron diferentes habilidades de abordaje con los adultos que permitió el despliegue de su rol como 'multiplicadores' de prácticas de crianza positiva, orientadas al empoderamiento de sus capacidades como agentes constructores de paz, esto permitió implementar en sus hogares acciones favorables al desarrollo humano de los niños y las niñas, que como punto de partida retoma las características particulares de cada grupo familiar.

3. El proceso de formación a las agentes educativas, a través del diplomado "Crianza para la Paz”, aportó a la cualificación de su quehacer pedagógico y profesional y dio apertura a las alternativas de intervención desde una perspectiva sistémica, construyendo colectivamente con los niños, niñas y sus familias abordajes de la primera infancia desde los diálogos generativos y la mirada apreciativa como recursos que posibilitan la participación intergeneracional y la creación y construcción de relaciones emergentes a la naturalización de la violencia. 
Investigación para la paz: perspectiva de paz desde la primera infancia y sus agentes relacionales

\section{Referencias}

Alvarado, L. y García, M. (2008). Características más relevantes del paradigma socio-crítico: su aplicación en investigaciones de educación ambiental y de enseñanza de las ciencias realizadas en el Doctorado de Educación del Instituto Pedagógico de Caracas. Sapiens. Revista Universitaria de Investigación, 9, 187-202.

Barudy, J. (2005). Los buenos tratos y la resiliencia infantil en la prevención de los trastornos del comportamiento. Recuperado de http: / /www.obelen.es/upload/383D.pdf

Barudy, J. y Dantagnan, M. (2005). Los Buenos Tratos de la Infancia Parentalidad, Apego y Resiliencia. Barcelona, España: GEDISA.

Bollinger, D. (1991). Sobre la escuela:entrevista a Pierre Bourdieu para el CNDP. Recuperado de https: / / www.studocu.com/es/document/universidad-de-cantabria/desarrollo-curricular-en-elambito-de-lo-social/apuntes/sobre-la-escuela-entrevista-a-p-bourdieu/1604420/view

Boza, M. (2011). El Paradigma Socio-Crítico. Recuperado de http://innomente.blogspot. com/2011/05/el-paradigma-socio-critico_19.html

Centro Internacional de Educación y Desarrollo Humano. (2004). Boletín $N^{\circ} 3$ Conexión Niñez: Espacio Informativo y de encuentro para Trabajadores de Campo en América Latina y el Caribe.

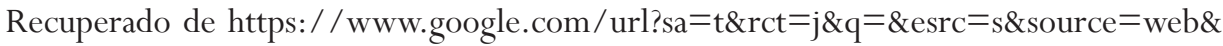
$\mathrm{cd}=1 \&$ ved $=2$ ahUKEwiV7s OUiITfAhUGrlkKHQGnBx4QFjAAegQIBRAC\&url=http \% 3A\%2F\%2Fwww.redprimerainfancia.org\%2Faa\%2Fimg_upload\%2F0b212a7b71568a8 d8c75d183a7feab2e\%2Fbolet_n1.pdf\&usg=AOvVaw3Xtf4QudMWgOb7e5EHOayZ

Concha, P.C. (2009). Teoría de los conflictos. Revista Paz y Conflictos, 60-81.

Fisas, V. (2011). Educar para la Paz. QUADERNS DE CONSTRUCCIÓ DE PAU, 20, 1-10.

Gallego, A. M. (2015). Participación infantil... Historia de una relación de invisibilidad. Revista Latinoamericana de Ciencias Sociales, Niñez y Juventud, 13 (1), 151-165.

Guerra, E. (2010). Las teorías sociológicas de Pierre Bourdieu y Norbert Elias: los conceptos de campo social y habitus. Estudios Sociológicos, XXVIII (83), 383-409.

Gómez, E.O. y Villa, J. (2014). Hacia un concepto interdisciplinario de la familia en la globalización. Justicia Juris, 10 (1), 11-20.

Gough, I. (2007). El enfoque de las capacidades de M. Nussbaum: un analisis comparado con nuestra teoría de las necesidades humanas. Madrid, España: Papeles de Relaciones Ecosociales y Cambio Global, no 100, Centro de Investigación para la Paz. 
Guasch i Garcia, M. y Domínguez, M.J. (2012). Estilo de socialización parental y fracaso. Recuperado de https://www.tdx.cat/bitstream/handle/10803/84052/Tesis\%20Ma. Jos\%E9\%20Dom\%EDnguez, \%2017-07-2012.pdf?sequence $=1$

Hernández, L.A. (2012). Empatía y altruismo en los procesos de alteridad de niños de educación primaria (tesis de maestria). Universidad Pedagogica Nacional. México D.F., México.

Ley 82 de 1993 (3 de noviembre), por la cual se expiden normas para apoyar de manera especial a la mujer cabeza de familia. Diario Oficial 41.101.

Ley 294 de 1996 (16 de julio), por la cual expide el artículo 42 de la constitución política. Diario Oficial No. 42.836.

Muñoz, M. (2009). ENTREVISTA A FEDERICO MAYOR ZARAGOZA. Revista Avances en supervisión educativa, (11), 1-10. Recuperado de https://avances.adide.org/index.php/ ase/article/view/404/476

Olivar, R.R. (2004). Inteligencia prosocial: educación de las emociones y valores. Barcelona, España: Universitat Autonoma de Barcelona.

Rodrigo, M., Martín, J., Cabrera, E. y Máiquez, M. (2009). Las Competencias Parentales en Contextos de Riesgo Psicosocial. Psychosocial Interventionn, 18 (2), 113-120.

Rodríguez, R. B. (2012). Martha Nussbaum: Las capacidades humanas y la vida buena. Recuperado de http://www.ieturolenses.org/revista_turia/index.php/actualidad_turia/marthanussbaum-las-capacidades-humanas-y-la-vida-buena

Salgado, S.V., Patiño, J. y Ospina, M. (2011). Ecoclubes una experiencia de socialización política con niños, niñas y jóvenes: el cuidado del ambiente como pre-texto para la formación y acción política. En H.F. Serna., S. V. Alvarado. y P. Botero. (Eds.), Experiencias Alternativas de acción política con participación de jóvenes en Colombia (pp. 115-139). Manizales, Colombia: Centro de Estudios Avanzados en Niñez y Juventud. 